\title{
Change in Mass of Spinning Black Holes w.r.t. the Angular Momentum
}

\author{
Dipo Mahto1, Anuradha Kumari² \\ ${ }^{1}$ Department of Physics, Marwari College, T. M. B. University, Bhagalpur, India \\ ${ }^{2}$ Department of Physics, T. M. B. University, Bhagalpur, India \\ Email: dipomahto@hotmail.com, anuphy1987@gmail.com
}

How to cite this paper: Mahto, D. and Kumari, A. (2018) Change in Mass of Spinning Black Holes w.r.t. the Angular Momentum. Journal of Modern Physics, 9, 1037-1042.

https://doi.org/10.4236/jmp.2018.95065

Received: March 4, 2018

Accepted: April 25, 2018

Published: April 28, 20188

Copyright (c) 2018 by authors and Scientific Research Publishing Inc. This work is licensed under the Creative Commons Attribution International License (CC BY 4.0).

http://creativecommons.org/licenses/by/4.0/

(c) (i) Open Access

\begin{abstract}
Mahto et al. have shown $\delta S \geq 0$ and $M^{2}=a^{*} J$ by using the first law of the black hole mechanics in the vacuum and Einstein mass-energy equivalence relation specially for spinning black holes. In the present paper, this work is extended to propose a model for the change in mass of the spinning black holes due to corresponding change in the angular momentum for maximum $\&$ half spin parameter of black holes $\left(a^{*}=1 \& 1 / 2\right)$ and calculated their values for different test black holes in XRBs and AGN. We have also shown that the change in mass of the spinning black holes due to corresponding change in the angular momentum for maximum spinning rate of black holes $\left(a^{*}=1\right)$ is double to that of the spinning black holes having spinning parameter $\left(a^{*}=\right.$ $1 / 2)$.
\end{abstract}

\section{Keywords}

Spinning Black Holes, Angular Momentum, Spinning Parameter

\section{Introduction}

Classically black holes are perfect absorbers, but do not emit anything; their physical temperature is absolute zero [1]. Quantum mechanically, however, there is a possibility that one of a particle production pairs in a black hole is able to tunnel the gravitational barrier and escapes the black hole's horizon. Thus it can radiate or evaporate particles [2]. The mass, area and surface gravity of the black hole mechanics have the same role as the energy, entropy and temperature in the ordinary laws of thermodynamics [3]. In 2006, B. Aschenbach has shown that the orbital velocity of a test particle is no longer a monotonic function of the orbit radius when the spin of the black hole is greater than 0.9953 , but displays a local minimum-maximum structure for radii smaller than 1.8 gravita- 
tional radii [4]. In 2007, Adel Bouchareb and Gerard Clement extended the Abbott-Deser-Tekin approach to the Computation of the Killing charge for a solution of topologically massive gravity (TMG) linearized around an arbitrary background and then applied to evaluate the mass and angular momentum of black hole solutions of TMG with non-constant curvature asymptotic [5]. In 2009, Richard B Larson suggested that in all cases, gravitational interactions with other stars or mass concentrations in a forming system play an important role in redistributing angular momentum and thereby enabling the formation of a compact object [6]. In 2013, Mahto et al. have shown $\delta S \geq 0$ to use the first law of the black hole mechanics in the vacuum and Einstein mass-energy equivalence relation specially for spinning black holes and established the relation $M^{2}=a^{*} J$ by entirely new methods [7]. All the works done as discussed above do not give the comparative study for the change in mass of the spinning black holes due to corresponding change in the angular momentum for half spin and maximum spin of the black holes.

In the present work, a model for the change in mass of the spinning black holes due to corresponding change in the angular momentum is proposed for maximum spinning rate of black holes $\left(a^{*}=1 / 2\right.$ and 1$)$.

\section{Theoretical Discussion}

The mass $(M)$, angular momentum $(/)$ and spin parameter $\left(a^{*}\right)$ of black holes are co-related by the following equation [7].

$$
M^{2}=a * J
$$

where $a^{*}$ is a constant called spin parameter of spinning black holes lying between -1 to +1 including zero of different test black holes [8] .

Now the equation is differentiated, we have

$$
\begin{aligned}
& 2 M \delta M=a^{*} \delta J \\
& \frac{\delta M}{\delta J}=\frac{a^{*}}{2 M}
\end{aligned}
$$

For spinning black holes having maximum spin $a^{*}=1 \quad$ [8]. Hence Equation (2) leads to

$$
\frac{\delta M}{\delta J}=\frac{1}{2 M}
$$

The above equation shows that the change in mass of the spinning black holes due to corresponding change in the angular momentum for maximum spinning rate of black holes.

For half spin parameter $\left(a^{*}=1 / 2\right)$, the Equation (2) becomes [9]

$$
\frac{\delta M}{\delta J}=\frac{1}{4 M}
$$

The above equation shows that the change in mass of the spinning black holes due to corresponding change in the angular momentum for half spin parameter of black holes. 


\section{Data in the Support for Mass of Black Holes and Sun}

There are two categories of black holes classified on the basis of their masses clearly very distinct from each other, with very different masses $M \sim 5-20 M_{\odot}$ for stellar-mass Black holes in X-ray binaries and $M \sim 10^{6}-10^{9.5} M_{\odot}$ for super massive black holes in Active Galactic Nuclei [8]. Mass of sun $\left(M_{\odot}\right)=1.99 \times 10^{30}$ $\mathrm{kg}[8]$.

On the basis of the data mentioned in the Section 3, the change in mass of different test spinning black holes due to corresponding change in angular momentum are calculated in XRBs and AGN to plot the graphs with the help of Equation (3) \& (4) as shown in Figures 1-3 of the Section 4.

\section{Result and Discussion}

To obtain the change in mass of the spinning black holes due to corresponding change in the angular momentum, the equation (1) is differentiated for maximum spinning rate of black holes $\left(a^{*}=1\right)$ and half spin parameter $\left(a^{*}=1 / 2\right)$ given by the Equation (3) \& (4) respectively.

After this, we have calculated the change in mass of the spinning black holes due to corresponding change in the angular momentum for maximum spinning rate of black holes $\left(a^{*}=1 \& a^{*}=1 / 2\right)$ in XRBs and AGN with the help of (3) \& (4).

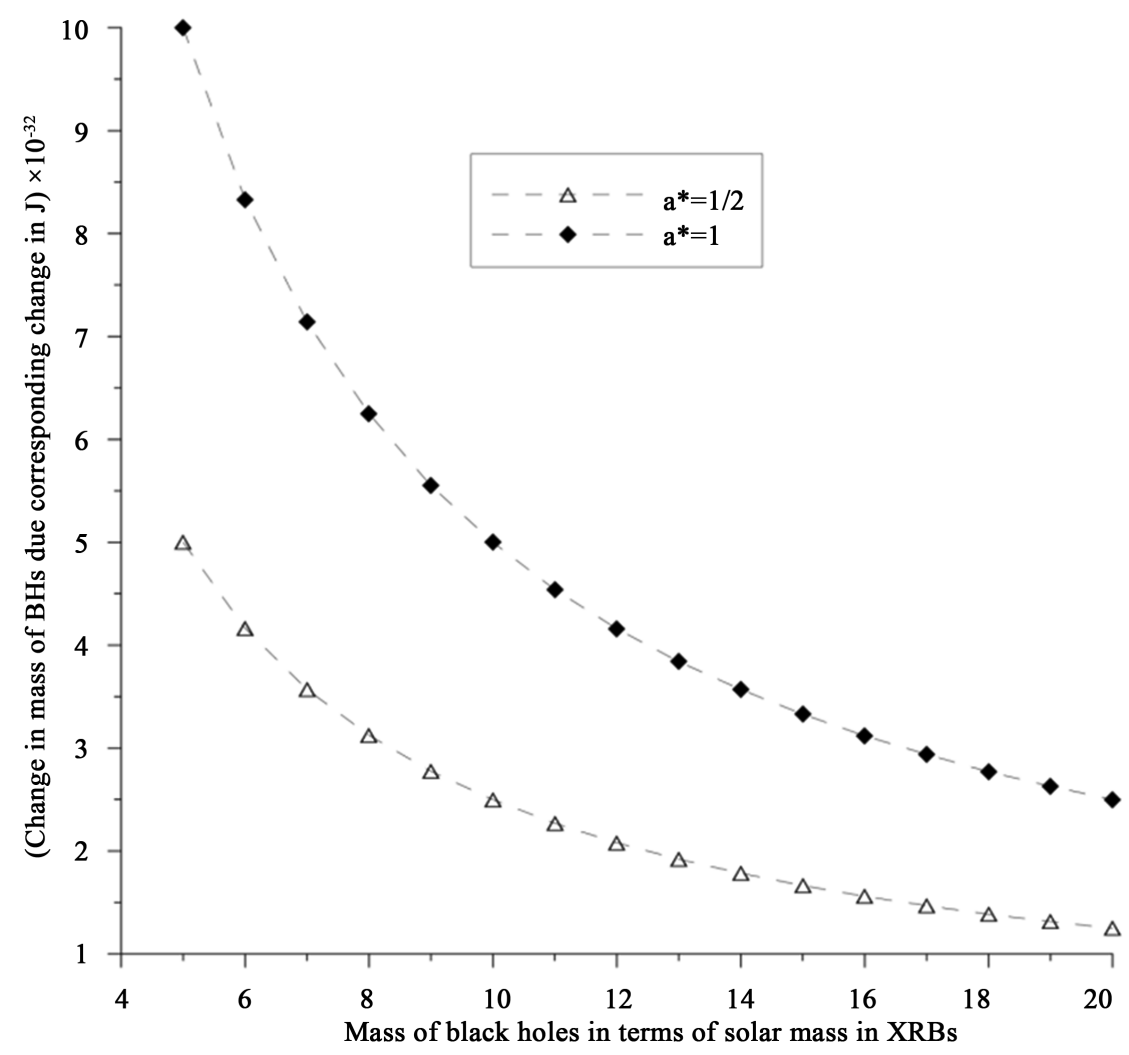

Figure 1. The graph plotted between the mass of spinning black holes and corresponding change in their angular momentum in XRBs with spin parameter $a^{\star}=1 / 2$ and 1 . 


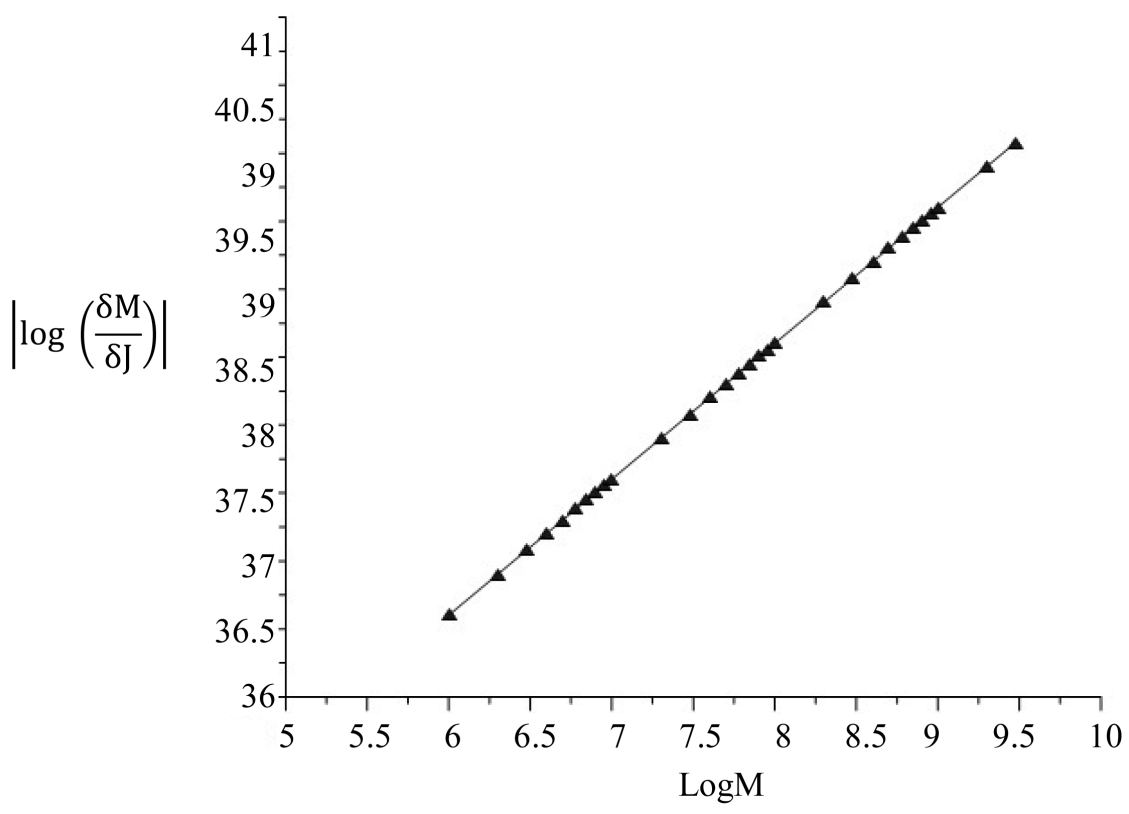

Figure 2. The graph plotted between the mass of spinning black holes and corresponding change in their angular momentum in AGN with spin parameter $a^{*}=1 / 2$ using logarithmic scale.

We have plotted the graph between the change in mass of the spinning black holes w.r.t. the change in the angular momentum for maximum spinning rate of black holes $\left(a^{*}=1\right)$ and spinning parameter $\left(a^{*}=1 / 2\right)(\delta M / \delta J)$ and corresponding value of the mass of black holes $(M)$. We also have shown that the change in mass of the spinning black holes due to corresponding change in the angular momentum for maximum spinning rate of black holes $\left(a^{*}=1\right)$ is greater than to that of the spinning black holes having spinning parameter $\left(a^{*}=1 / 2\right)$ in $\mathrm{XRBs}$ and shows that the change in mass of the spinning black holes w.r.t. the change in the angular momentum for maximum spinning rate of black holes $\left(a^{*}\right.$ $=1)$ is twice times to that of spinning of black holes with spinning parameter $\left(a^{*}\right.$ $=1 / 2$ ). This fact is also clear from the comparative study from the Figure 1 for $\mathrm{XRBs}$, while in the case of AGN, this change is quite different regarding the maximum and half integral spin as clear from the Figure $2 \&$ Figure 3.

Figure 2 \& Figure 3 show the graph plotted between the mass of spinning black holes and corresponding change in their angular momentum in AGN with spin parameter $a^{*}=1 / 2$ and $a^{*}=1$ respectively. From both the graphs, it is clear that the change in mass of the spinning black holes w.r.t. the change in the angular momentum for maximum spinning rate of black holes $\left(a^{*}=1\right)$ fluctuates with certain values on increasing the value of the mass of the spinning black holes, while for the same mass of black holes with spin parameter $\left(a^{\star}=1 / 2\right)$, there is uniform variation in mass of the spinning black holes w.r.t. the change in the angular momentum on increasing the value of the mass of the spinning black holes.

From the graph in the Figure 3, it is obvious that the change in mass of 


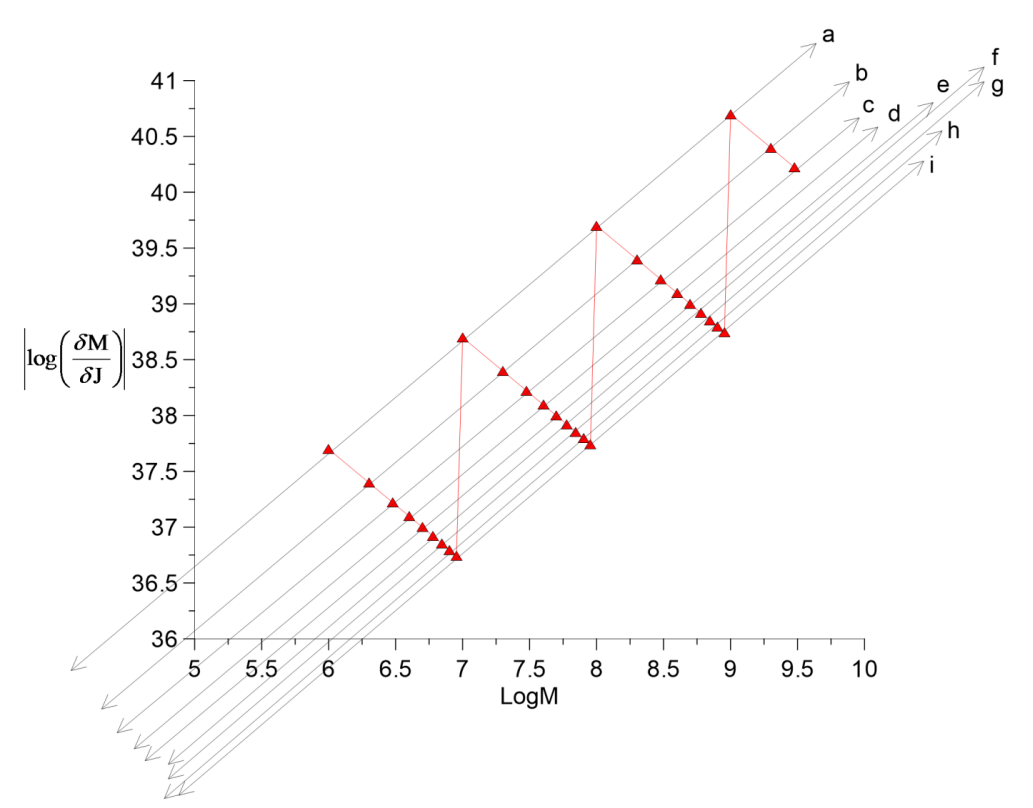

Figure 3. The graph plotted between the mass of spinning black holes and corresponding change in their angular momentum in AGN using logarithmic scale explaining complex nature of spinning black holes with spin parameter $a^{*}=1$.

spinning black holes and corresponding change in their angular momentum in AGN with spin parameter $a^{*}=1$ decreases up to a certain value of the mass with the increasing the mass of the spinning black holes and then rapidly increases with a certain value These variations are repeated in periodic manner as shown in Figure 3. On the basis of observation of the graph in the Figure 3, the black holes can be categorized regarding their same order of mass or radius of event horizon as discussed below:

The spinning black holes of mass:

1) $\left(1 \times 10^{6} M_{\odot}, 1 \times 10^{7} M_{\odot}, 1 \times 10^{8} M_{\odot}, 1 \times 10^{9} M_{\odot}\right)$-line a

2) $\left(2 \times 10^{6} M_{\odot}, 2 \times 10^{7} M_{\odot}, 2 \times 10^{8} M_{\odot}, 2 \times 10^{9} M_{\odot}\right)$-line b

3) $\left(3 \times 10^{6} M_{\odot}, 3 \times 10^{7} M_{\odot}, 3 \times 10^{8} M_{\odot}, 3 \times 10^{9} M_{\odot}\right)$-line c

4) $\left(4 \times 10^{6} M_{\odot}, 4 \times 10^{7} M_{\odot}, 4 \times 10^{8} M_{\odot}, 4 \times 10^{9} M_{\odot}\right)$-line d

5) $\left(5 \times 10^{6} M_{\odot}, 5 \times 10^{7} M_{\odot}, 5 \times 10^{8} M_{\odot}, 5 \times 10^{9} M_{\odot}\right)$-line e

6) $\left(6 \times 10^{6} M_{\odot}, 6 \times 10^{7} M_{\odot}, 6 \times 10^{8} M_{\odot}, 6 \times 10^{9} M_{\odot}\right)$-line $\mathrm{f}$

7) $\left(7 \times 10^{6} M_{\odot}, 7 \times 10^{7} M_{\odot}, 7 \times 10^{8} M_{\odot}, 7 \times 10^{9} M_{\odot}\right)$-line g

8) $\left(8 \times 10^{6} M_{\odot}, 8 \times 10^{7} M_{\odot}, 8 \times 10^{8} M_{\odot}, 8 \times 10^{9} M_{\odot}\right)$-line h

9) $\left(9 \times 10^{6} M_{\odot}, 9 \times 10^{7} M_{\odot}, 9 \times 10^{8} M_{\odot}, 0 \times 10^{9} M_{\odot}\right)$-line $\mathrm{i}$

When the graph is plotted for each category in the same graph paper, nine parallel lines are obtained. When the results obtained from our research work is compared with that of the spinning black holes with spin $a^{*}=1 / 2$, we see that the change in mass of the spinning black holes w.r.t. the change in the angular momentum for maximum spinning rate of black holes $\left(a^{*}=1\right)$ is quite different to that of the spinning black holes with spin parameter $\left(a^{*}=1 / 2\right)$. Hence, we may conclude that the spinning parameters of black holes are mainly responsible for the identification and characterization of black holes. 


\section{Conclusions}

In the study of present research paper, we can draw the following conclusions:

1) The change in mass of the spinning black holes due to corresponding change in the angular momentum for the black holes of maximum spin is double to that of the spinning black holes of half spin parameter and decreases with increasing the mass of black holes in XRBs.

2) The change in mass of the spinning black holes w.r.t. the change in the angular momentum for the black holes of maximum spin is quite different to that of the spinning black holes with half spin parameter in AGN.

3) The spinning parameter is mainly responsible for the identification and characterization of black holes.

\section{Acknowledgements}

The authors are very grateful to reviewers to find out the errors in the original manuscript and providing the constructive suggestions. This paper is also devoted in memory of great scientist Stephen Hawking (March 14, 2018).

\section{References}

[1] Wald, R.M. (2001) Living Reviews in Relativity, 4, 6. https://doi.org/10.12942/lrr-2001-6

[2] Triyanta, T. and Bowaire, A.N. (2013) Journal of Mathematical and Fundamental Sciences, 45, 114-123. https://doi.org/10.5614/j.math.fund.sci.2013.45.2.2

[3] Bardeen, J.M., Carter, B. and Hawking, S.W. (1973) Communications in Mathematical Physics, 31, 161-170. https://doi.org/10.1007/BF01645742

[4] Aschenbach, B. (2006) Chinese Journal of Astronomy and Astrophysics, 10, 24.

[5] Adel, B. and Gerard, C. (2007) Classical and Quantum Gravity, 24, 5581-5594. https://doi.org/10.1088/0264-9381/24/22/018

[6] Richard, B.L (2010) Reports on Progress in Physics, 73, Article ID: 014901, 14 p.

[7] Mahto, D., Kumari, A. and Singh, K.M. (2013) International Journal of Engineering and Innovative Technology, 3, 270-273.

[8] Narayan, R. (2005) Black Holes in Astrophysics. arXiv:gr-qc/0506078 V1.

[9] Mahto, D., Singh, A.K. and Kumari, N. (2016) International Journal of Astronomy and Astrophysics, 6, 328-333. https://doi.org/10.4236/ijaa.2016.63027 